\title{
ANÁLISE DE CONTAMINANTES DE FASE LÍQUIDA NÃO AQUOSA (NAPLS) POR APLICAÇÃO DO MÉTODO ELETROMAGNÉTICO INDUTIVO (EM)
}

\author{
César Augusto Moreira ${ }^{1}$ e João Carlos Dourado² \\ Recebido em 24 novembro, 2004 / Aceito em 21 junho, 2005 \\ Received on November 24, 2004 / Accepted on June 21, 2005
}

\begin{abstract}
The improper discarding of industrial residues in the soil has caused damages to the environment and to the human health. The identification and diagnosis of contaminated areas are necessary procedure of that it is possible to take measured by containment of the polluting source and posterior remediation. The geophysical techniques can assist the characterization of the ground subsurface, definition of the contamination plumesize, selection of sampling points and pumping wells. This work presents some results from the application of the Inductive Electromagnetic method in an industrial area contaminated by benzene, toluene, xylene (BTX) and dichloroethene. The results indicate two migrations of the patterns for the subsurface contaminants. A horizontal trend of migration extends up to $15 \mathrm{~m}$ depth and a vertical trend of migration in the vertical plan enters 15 to $60 \mathrm{~m}$ depth. The divergent trends of migration are conditioned by density of the composites that constitute the plume of contamination, or either, a floating interval LNAPL (BTX), overlapped to an interval DNAPL (1,2 dichloroethene).
\end{abstract}

Keywords: Inductive Electromagnetic, Contamination plume, BTX, LNAPL, DNAPL.

RESUMO. 0 descarte indevido de resíduos industriais no solo tem causado danos ao meio ambiente e à saúde humana. A identificação e o diagnóstico de áreas contaminadas é um procedimento necessário afim de que seja possível tomar medidas de contenção da fonte poluidora e posterior remediação. As técnicas geofísicas podem auxiliar na caracterização do meio geológico, definição das dimensões da pluma de contaminação, seleção de pontos para locação de poços de monitoramento e de bombeamento. Este trabalho apresenta os resultados da aplicação do método Eletromagnético Indutivo em uma área industrial contaminada por benzeno, tolueno, xileno (BTX) e dicloroetano. Os resultados indicam dois padrões de migração dos contaminantes em subsuperfície. Uma tendência de migração no plano horizontal até 0 nível de $15 \mathrm{~m}$ de profundidade e uma tendência de migração no plano vertical entre 15 e $60 \mathrm{~m}$ de profundidade. As tendências de migração divergentes estão condicionadas a densidade dos compostos que constituem a pluma de contaminação, ou seja, um intervalo LNAPL sobrenadante (BTX), sobreposto a um intervalo DNAPL (1,2 dicloroetano).

Palavras-chave: Eletromagnético Indutivo, Pluma de contaminação, BTX, LNAPL, DNAPL.

\footnotetext{
1Pós-graduação em Geociências em Meio Ambiente - IGCE - UNESP - Campus de Rio Claro Rua 10, n. 2527 - Bairro Santana - 13500-230 Rio Claro, SP, Brasil. E-mail: cesargeologia@yahoo.com.br

${ }^{2}$ Departamento de Geologia Aplicada - IGCE - UNESP - Campus de Rio Claro, Av. 24-A n. 1515 - Bela Vista - Caixa Postal 178, 13506-900 Rio Claro, SP, Brasil. E-mail: jdourado@rc.unesp.br
} 


\section{INTRODUÇÃo}

0 uso crescente de derivados de petróleo em processos industriais proporciona a geração de uma diversidade de produtos cada vez mais utilizados pela sociedade atual. Uma das conseqüências negativas do uso e manipulação de hidrocarbonetos é a geração de resíduos e efluentes altamente poluidores.

Os hidrocarbonetos são denominados compostos de fase líquida não aquosa (NAPLs), cujas diferenças nas propriedades físicas e químicas resultam na imiscibilidade em água (Newell et al., 1995). Quanto à densidade, os compostos de NAPLs podem ser divididos em duas classes:

- Compostos de Fase Líquida Leve Não Aquosa - LNAPL, caracterizados por serem mais leves que a água. Tais compostos são representados na área contaminada por benzeno, tolueno e xileno.

- Compostos de Fase Líquida Densa Não Aquosa - DNAPL, caracterizados por serem mais densos que a água. Esta fase contaminante é representada na área por 1, 2 dicloroetano.

A destinação destes resíduos tem sido um dos principais problemas ambientais da atualidade, pois muitas vezes não existem opções de reutilização e o transporte e disposição em locais adequados, demanda elevados investimentos. Procedimentos inadequados resultam em ações danosas ao meio ambiente, como 0 descarte indevido, com graves conseqüências tanto ao solo quanto aos recursos hídricos subjacentes.

Desta maneira, são necessárias técnicas de investigação com 0 intuito de avaliar a extensão da contaminação e os impactos causados, bem como sua evolução ao longo do tempo.

A aplicação do método Eletromagnético Indutivo (EM) em estudos ambientais reside na possibilidade de determinação da condutividade aparente do terreno para diversas profundidades. As principais vantagens deste método são a facilidade de aquisição de dados, realização de leituras em diversas profundidades, versatilidade do equipamento em campo e a possibilidade de varredura de grandes áreas num curto espaço de tempo (McNeill, 1980a; EPA, 1993; Goldstein et al., 1990). Estas vantagens são traduzidas em rapidez e baixos custos.

Uma das principais limitações desta técnica é o ruído produzido pela interação do campo eletromagnético gerado pelo equipamento, com estruturas metálicas, fios de alta tensão tambores metálicos e outras estruturas (McNeill, 1980a). Esta interação resulta em valores de condutividade aparente incoerentes, 0 que torna 0 uso desta técnica limitado em centros urbanos, instalações industriais, entre outros.

Este trabalho discute os resultados obtidos no mapeamento de uma pluma de contaminação constituída por hidrocarbonetos, por meio do método EM, com o objetivo de analisar o comportamento dos compostos frente à dinâmica no meio geológico.

\section{MATERIAIS E MÉTODOS Histórico da Área de Estudos}

A área de estudos está localizada no distrito industrial do município de Araras, estado de São Paulo, Brasil (Fig. 1). 0 local foi ocupado por duas indústrias químicas entre 1981 e 1992. Dentre os produtos fabricados estão o dieloxalato, acetato de etila, 4amino-N-2-tiozolil-benzeno-sulfonamida, acetopiruvato de metila, 5-metil-3-carboxiamidoxazol, e dentre os solventes utilizados durante o processo estão 0 1,2 dicloroetano e o tolueno (CETESB, 1997). Os solventes eram reutilizados após passarem por colunas de destilação, e os efluentes gerados eram infiltrados diretamente no solo, em dois poços existentes na área. Este procedimento foi utilizado entre 1981 e 1988 (CETESB, 1997).

Em 1988 foi constatada a contaminação solo e da água subterrânea por 1,2 dicloroetano, benzeno, tolueno, xileno, sódio e cloreto. Em 1990 foi iniciado o monitoramento da contaminação pela Agência de Proteção Ambiental do Estado de São Paulo (CETESB), por meio de diversos poços de monitoramento instalados na área industrial e adjacências.

\section{Caracterização da Área de Estudos}

0 meio geológico é caracterizado por siltitos argilosos pertencentes à formação Tatuí, representados no local por solo vermeIho escuro argiloso, com matriz de fração silte entre 1 e 3 metros de profundidade e matriz de fração argila entre 3 a 13 metros de profundidade, com transição gradual entre os níveis. 0 nível freático está situado entre 10,7 e 14,1 metros de profundidade, com oscilações máximas entre 7,9 a 14,9 metros. 0 fluxo da água subterrânea nas proximidades dos pontos de infiltração de resíduos é para sudoeste. A condutividade hidráulica varia de 4, $4 \times 10^{-7}$ a $7,0 \times 10^{-7} \mathrm{~cm} / \mathrm{s}$ (CETESB, 1997; Aquino, 2000).

\section{Método Eletromagnético Indutivo}

0 método EM determina a condutividade elétrica dos materiais em subsuperfície utilizando o princípio da indução eletromagnética em baixo número (low induction number) (Benson \& Stubben, 1995).

Este método tem como procedimento básico a passagem de uma corrente elétrica alternada em uma bobina transmissora, que 


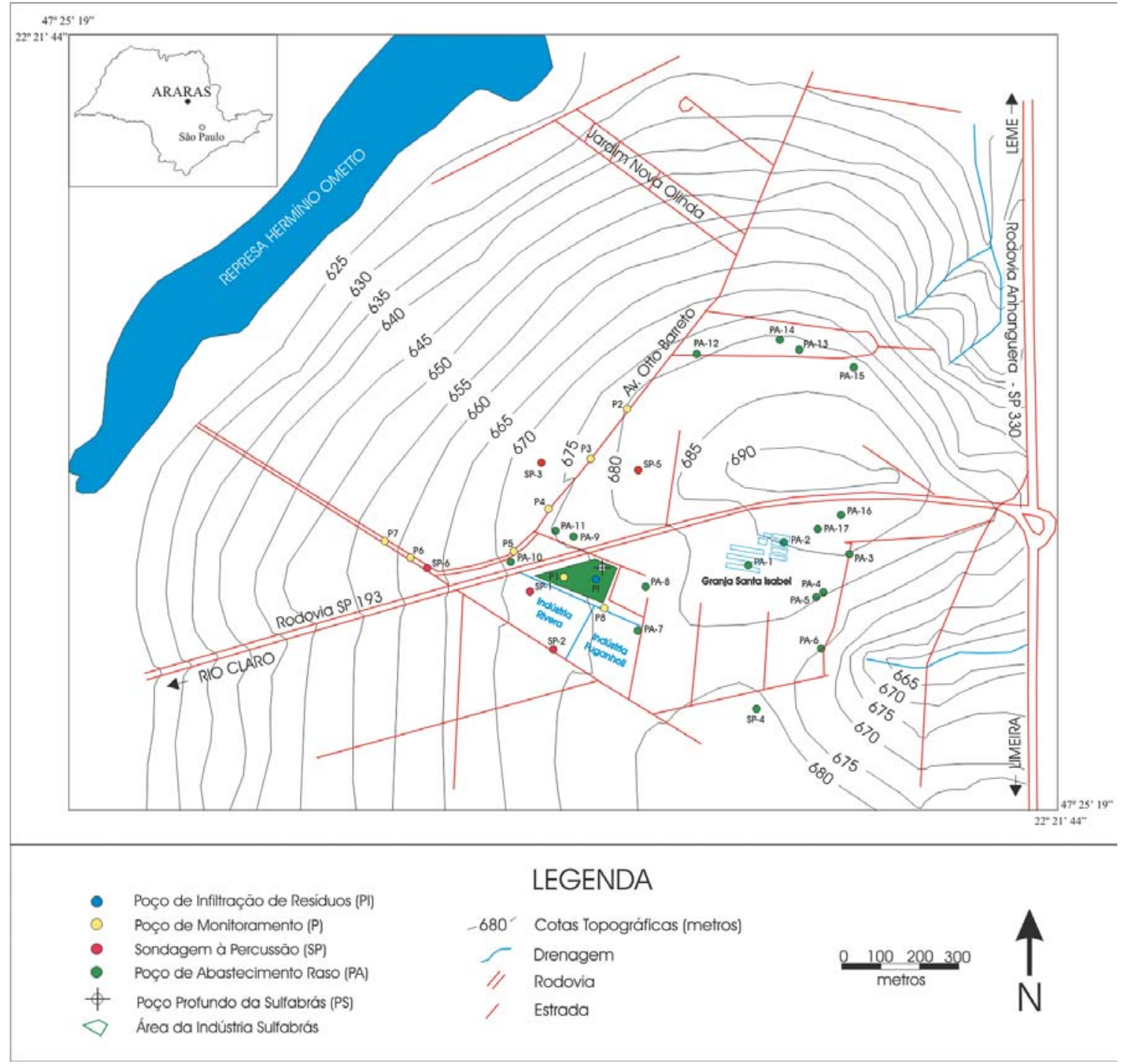

Figura 1 - Mapa de Localização.

resulta em um campo eletromagnético primário. A existência deste campo em um meio condutor induz correntes secundárias no subsolo, que por sua vez produzem um campo eletromagnético secundário (Fig. 2).

Uma bobina receptora posicionada dentro da área de influência dos campos primário e secundário capta a corrente induzida pela soma dos campos gerados. As diferenças de intensidade e fase entre os campos primário e secundário podem revelar a presença de corpos condutores.

A condutividade elétrica $(\sigma)$ do meio varia com 0 tipo de solo ou rocha, porosidade, permeabilidade, grau de saturação e também em função das propriedades eletroquímicas dos fluidos de preenchimento de poros, que em muitos casos é o fator domi- nante (Sheriff, 1989; McNeill, 1980b).

0 equipamento utilizado neste trabalho foi 0 Sistema Eletromagnético EM 34-3 modelo XL, fabricado pela Geonics Ltd. Canadá. Este equipamento é calibrado para leituras diretas de condutividade aparente por meio da expressão (equação 1):

$$
\sigma_{a}=\frac{4}{(2 \pi f) \mu s^{2}}\left(\frac{H s}{H p}\right)
$$

sendo $H s$ campo magnético secundário e $H p$ o campo magnético primário; $\sigma_{a}$ é a condutividade aparente, em siemens por metro; $\mu$ é a permeabilidade magnética, em henry por metro; $f$ é a freqüência em hertz e $s$ é o espaçamento entre as bobinas, em metros. 


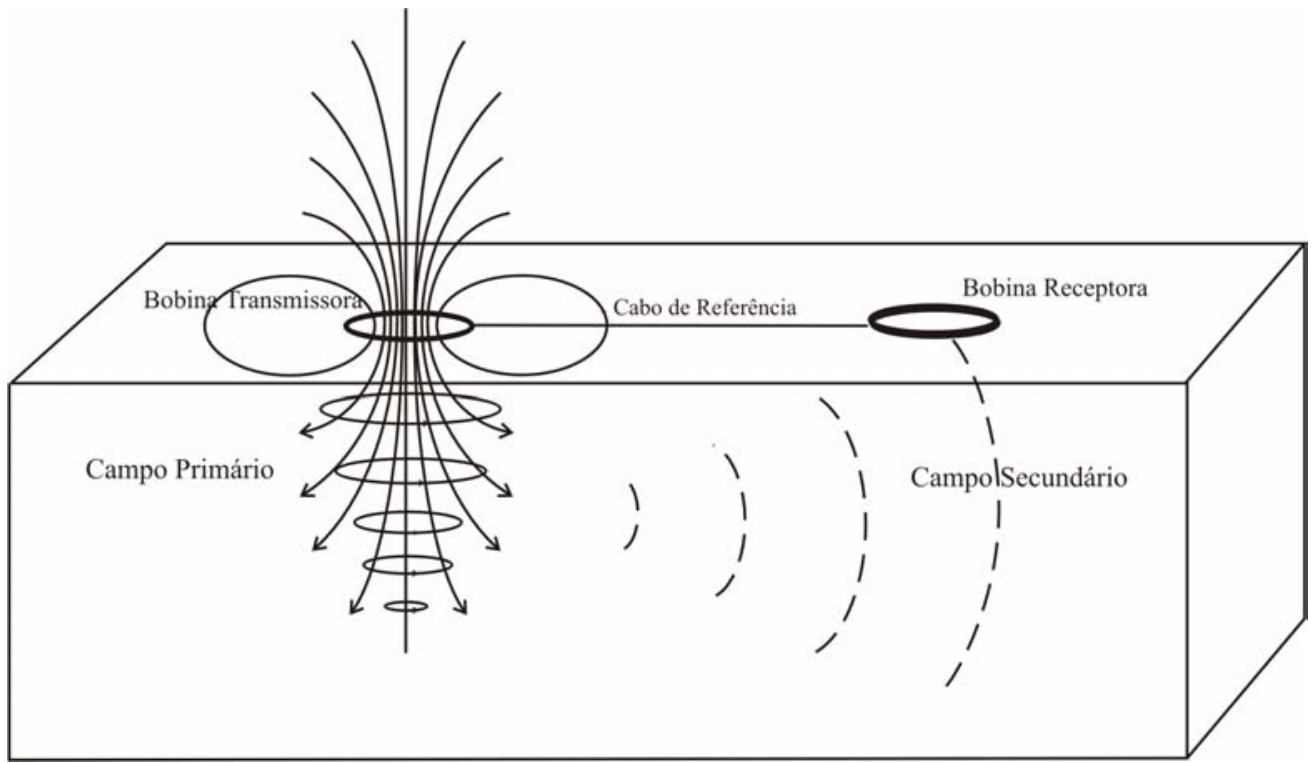

Figura 2 - Princípio do Método Eletromagnético Indutivo.

A variação na distância e na posição das bobinas permite a determinação da condutividade aparente para diversas profundidades. Neste trabalho foram utilizados os cabos de referência de 10 e 40 metros, que em conjunto com a variação na posição das bobinas permitiu leituras em quatro profundidades (Tab. 1).

Tabela 1 - Profundidades máximas de investigação em relação ao cabo de referência e a posição das bobinas (McNeill, 1980a).

\begin{tabular}{|c|c|c|}
\hline $\begin{array}{c}\text { Comprimento do } \\
\text { Cabo de Referência }(\mathrm{m})\end{array}$ & $\begin{array}{c}\text { Posição das } \\
\text { Bobinas }\end{array}$ & $\begin{array}{c}\text { Profundidade máxima } \\
\text { de Investigação }(\mathrm{m})\end{array}$ \\
\hline \multirow{2}{*}{10} & vertical & 7,5 \\
& horizontal & 15 \\
\hline \multirow{2}{*}{40} & vertical & 30 \\
& horizontal & 60 \\
\hline
\end{tabular}

\section{Aquisição e Processamento}

Os dados foram adquiridos com técnica de caminhamento eletromagnético, conforme proposto por Greenhouse \& Slaine (1986), com espaçamento médio de 20 metros entre pontos de leitura.

As linhas de caminhamento eletromagnético foram definidas com base em análises químicas da água subterrânea, levantamentos geofísicos preliminares e nos acessos disponíveis (CETESB, 1997; Aquino, 2000).

Foram realizadas 160 leituras de condutividade aparente para cada uma das profundidades máximas de investigação possíveis para o equipamento utilizado (Tab. 1).

Os dados foram interpolados por interpolação pelo método do inverso do quadrado da distância, por meio do programa SURFER, versão 8, da Golden Software - Estados Unidos. Durante o processamento foram excluídos alguns pontos de leitura, por apresentarem valores discrepantes, decorrentes da proximidade de transformadores elétricos, cruzamento de linhas de alta tensão, motores elétricos em funcionamento, dentre outros.

Os mapas de condutividade aparente para profundidades máximas de 7.5, 15, 30 e 60 metros são apresentados nas Figuras $3 \mathrm{~A}$ a $3 \mathrm{D}$.

\section{RESULTADOS}

A análise dos mapas de condutividade aparente indica que a pluma de contaminação atinge tanto a área da indústria quanto às áreas adjacentes.

Os mapas de condutividade aparente para as profundidades máximas de 7,5 e 15 metros (Figs. 3A e 3B) indicam que o ponto central da anomalia de condutividade esta associada ao poço de infiltração de resíduos. Desta forma, a anomalia de condutividade obtida está associada à pluma de contaminação.

A área de abrangência da pluma de contaminação para as profundidades de até 7,5 e 15 metros é muito superior a área de abrangência para as profundidades máximas de 30 e 60 metros (Figs. 3C e 3D).

0 mapa da pluma para as profundidades de até 7,5 e 15 metros resulta num corpo com formato cônico, com diâmetro de área basal superior a sua altura (fig. 4). Por outro lado, a integração 

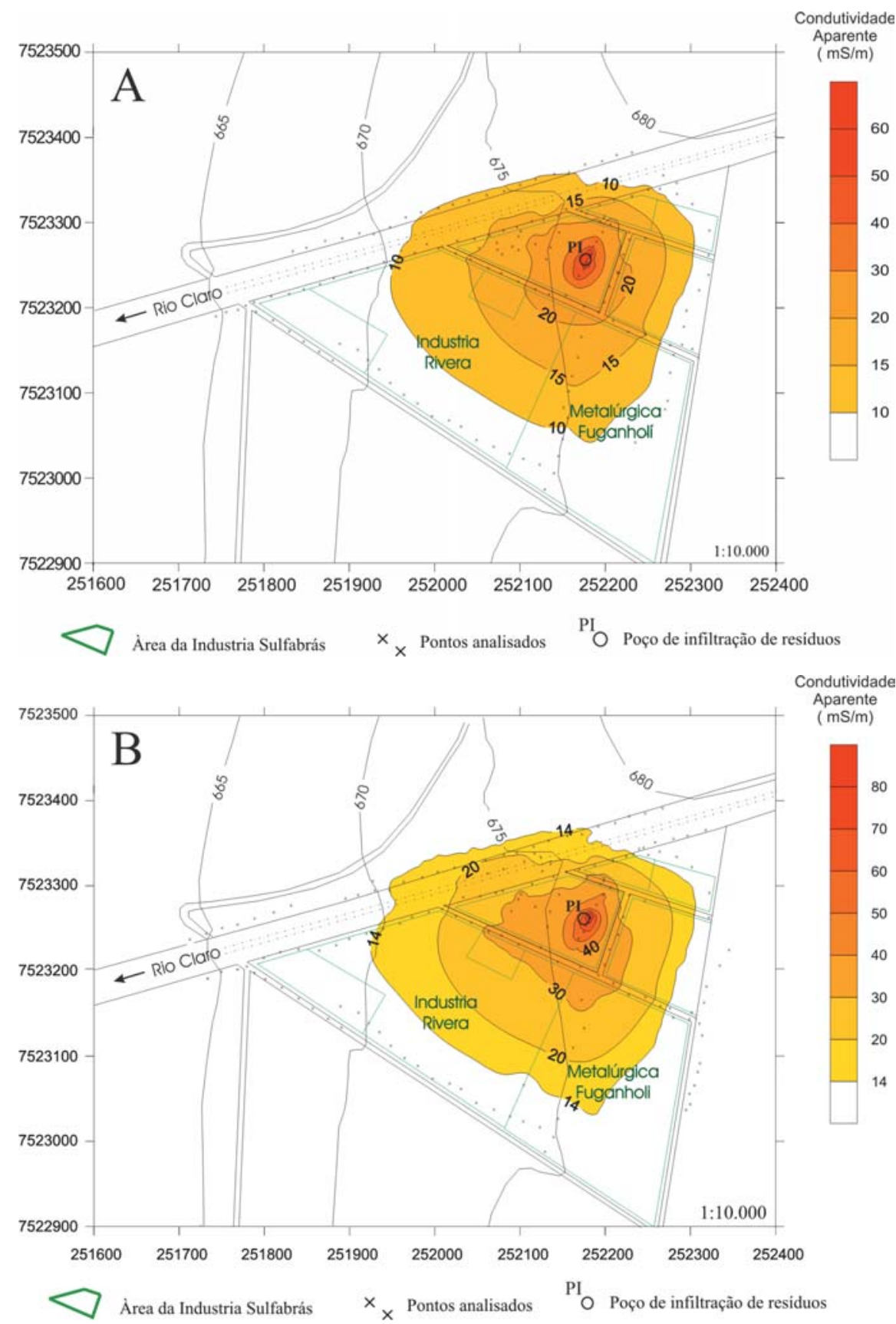

Figura 3 (A) e (B)

dos mapas para as profundidades de até 30 e 60 metros resulta num corpo com formato de cone invertido com diâmetro de área basal inferior a sua altura (fig. 4).

\section{DISCUSSÃO E CONCLUSÕES}

A detecção de anomalia de alta condutividade em profundidade foi possível devido à presença de compostos inorgânicos - sódio e cloreto - associados às frações degradadas dos compostos orgânicos, cujo produto resultante possui condutividade elétrica menor que o meio geológico. Nobes (1996) relata que a presença de contaminantes na zona saturada altera a condutividade elétrica do meio, que resulta em contraste com meio não contaminado em redor.

A degradação de hidrocarbonetos por atividade bacteriana 


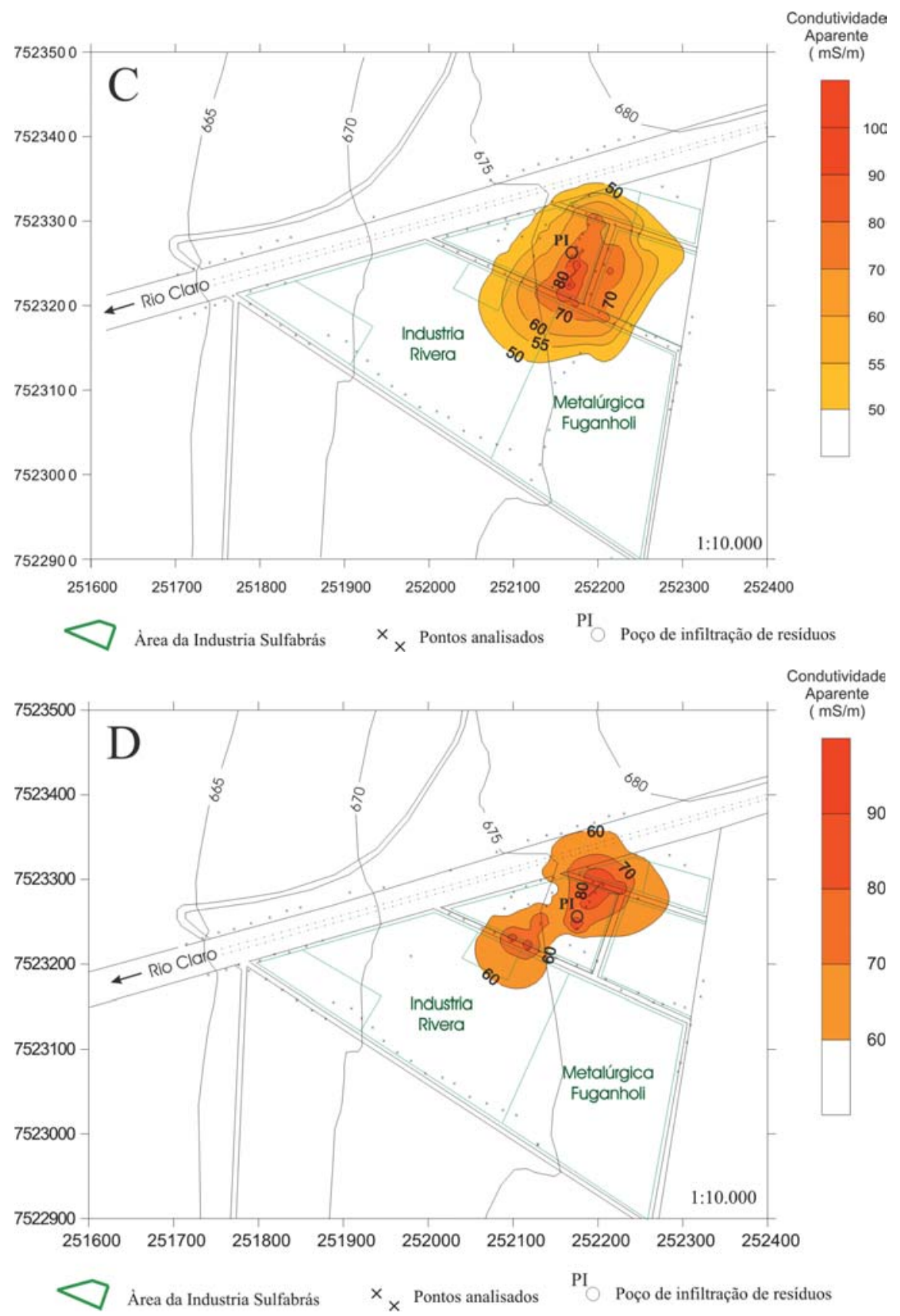

Figura 3 (C) e (D)

Figura 3 - Mapas de condutividade aparente para as profundidades máximas de 7,5 m (A), $15 \mathrm{~m}$ (B), $30 \mathrm{~m}$ (C) e $60 \mathrm{~m}$ (D).

na zona vadosa e no nível freático produz ácidos carbônicos e orgânicos, que contribuem com a dissolução dos materiais que comportam o aqüífero (McMahon et al., 1995). 0 aumento de sólidos totais dissolvidos (TDS) aumenta a condutividade hidráulica do aqüífero (Benson \& Stubben 1995).

Após a liberação no ambiente, os compostos de LNAPLs ten- dem a se a infiltrar no solo por ação da força da gravidade. Ao atingir a franja capilar, os compostos de LNAPL passam a ser mover lateralmente, sob a forma de fase livre ao longo da camada superior da zona saturada devido à ação da gravidade e de forças de capilaridade (Newell et al., 1995).

A migração de LNAPL em subsuperfície é controlada por 


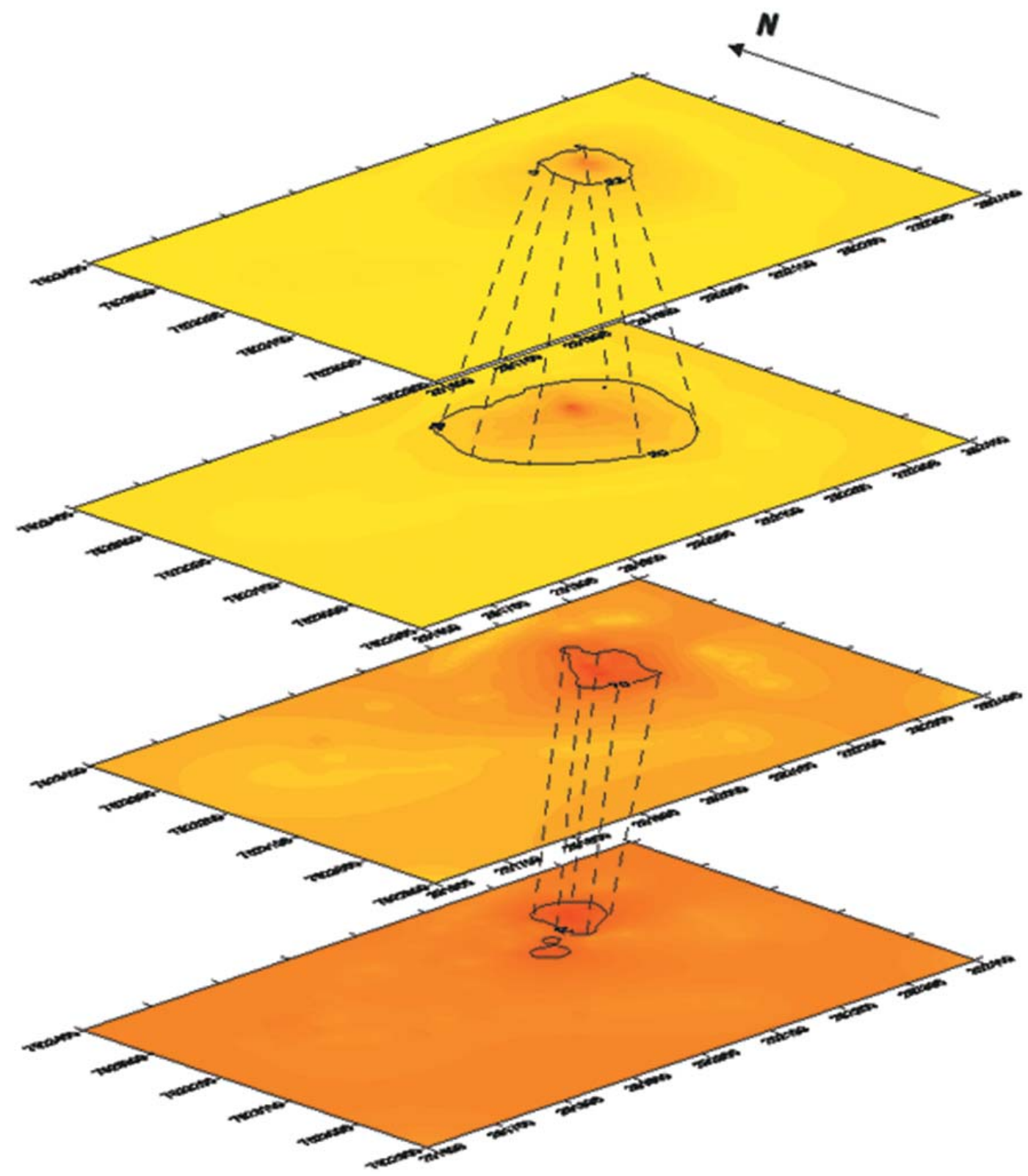

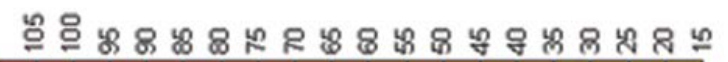

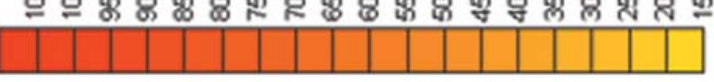

condutividade $\mathrm{mS} / \mathrm{m}$

Figura 4 - Integração dos mapas de condutividade aparente. Os primeiros dois níveis correspondem às profundidades de 7,5 e 15 metros e os últimos dois níveis correspondem às profundidades de 30 e 60 metros.

chuvas, variações sazonais e pelo gradiente hidrogeológico, associada ao fluxo d'água subterrânea (Daniels et al., 1994).

Segundo este raciocínio, as anomalias de condutividade detectadas nas profundidades máximas de 7,5 e 15 metros podem estar associadas a compostos de LNAPL, caracterizados por uma elevada migração horizontal associada ao fluxo da água subterrânea.

Os compostos de DNAPL tendem a migrar pelo interior da zona saturada por ação da gravidade e, eventualmente, são exauridos por processos de saturação residual. Contudo, caso este 
processo não consuma o DNAPL completamente, o composto continuará a migrar verticalmente até ser retido em zonas de baixa permeabilidade (Hulling \& Weaver, 1991).

Assim, as anomalias de condutividade detectadas nas profundidades máximas de 30 e 60 metros podem estar associadas a compostos de DNAPL que não foram consumidos na zona saturada, possivelmente devido as grandes quantidades liberadas, e que adquiriram a migração vertical.

\section{AGRADECIMENTOS}

Este trabalho foi realizado com o apoio da Agência Nacional do Petróleo - ANP e da Financiadora de Estudos e Projetos - FINEP, por meio do Programa de Recursos Humanos para o Setor de Petróleo e Gás - PRH-05-ANP/MCT.

Os autores agradecem ao técnico Francisco Manuel Garcia Barrera, do Departamento de Geologia Aplicada, UNESP - Rio Claro, pelo auxílio na execução dos trabalhos de campo.

\section{REFERÊNCIAS}

AQUINO WF. 2000. Métodos Geofísicos Eletromagnéticos aplicados ao diagnóstico da contaminação de Solo e das Águas Subterrâneas em área de Infiltração de Resíduos Industriais. pp. 121, Dissertação, Universidade de São Paulo.

BENSON AK \& STUBBEN MA. 1995. Interval resistivities and very low frequency electromagnetic induction - an aid to detecting groundwater contamination in space and time: a case study. Environmental Geosciences, 2: 74-84.

CETESB - COMPANHIA DE TECNOLOGIA DE SANEAMENTO AMBIENTAL. 1997. Relatório do levantamento geofísico para diagnóstico da contaminação da indústria Sulfabrás, CETESB, São Paulo, 91 p.

DANIELS JJ, ROBERTS R \& VENDI M. 1994. Ground Penetrating Radar for the detection of liquid contaminants. Journal of Applied Geophysics, 33: 195-207.

EPA - ENVIRONMENTAL PROTECTION AGENCY. 1993. Use of Airborne, Surface, and Borehole Geophysical Techniques at Contaminated Sites. Washington, chapter 1: 4-6.

GOLDSTEIN NE, BENSON SM \& ALUMBAUGH D. 1990. Saline Groundwater Plume Mapping with Electromagnetics. Geotechnical and Environmental Geophysics, Investigations in Geophysics, S.E.G., vol. II, 5: $17-27$

GREENHOUSE JP \& SLAINE DD. 1986. Geophysical modelling and mapping of contamined groundwater around three waste disposal sites in southern Ontario. Canadian Geotechnical Journal, vol. 23, p. 372-384.

HULLING SG \& WEAVER JW. 1991. Dense Nonaqueous Phase Liquids. Ground Water Issue, Environmental Protection Agency, Oklahoma, 21 p.

MCMAHON PB, VROBLESKY DA, BRADLEY PM, CHAPELLE FH \& GULLER CD. 1995. Evidence of enhance mineral dissolution in organic acidrich shallow ground water. Ground Water, 33: 207-216.

McNEILL J. 1980a. Electromagnetic Terrain Conductivity Measurement at Low Induction Numbers. Technical Note TN - 6, p. 6 - 15, 1980a. 〈http://www.geonics.com〉. May/2003.

McNEILL J. 1980b. Electrical Conductivity of Soils and Rocks. Technical Note TN - 5, pp. 21, 1980b. 〈http://www.geonics.com〉. May/2003.

NEWELL CJ, ACREE SA, ROSS RR \& HULLING SG. 1995. Light Nonaqueous Phase Liquids. Ground Water Issue, Environmental Protection Agency, Oklahoma, pp. 25.

NOBES DC. 1996. Troubled Waters: environmental applications of electrical and electromagnetic methods. Surveys in Geophysics 17: 393454.

SHERIFF RE. 1989. Geophysical Methods, Prentice Hall, New Jersey, pp. 175-201.

\section{NOTAS SOBRE OS AUTORES}

César Augusto Moreira. Bacharel em Geologia pelo Instituto de Geociência e Ciências Exatas (IGCE) da Universidade Estadual Paulista (UNESP) em 2003. Atualmente é aluno do programa de Pós-Graduação em Geociências e Meio Ambiente do Instituto de Geociências e Ciências Exatas - UNESP - Campus de Rio Claro.

João Carlos Dourado. Bacharel em Geologia pelo Instituto de Geociências da Universidade de São Paulo (USP) em 1977. Doutor em Geociências pela UNESP em 1997. Trabalhou com geofísica aplicada no Instituto de Pesquisas Tecnológicas do Estado de São Paulo de 1977 a 1997. Atualmente é professor assistente doutor do Departamento de Geologia Aplicada do IGCE-UNESP onde atua desde 1997. Trabalha com métodos geofísicos aplicados à Hidrogeologia, Meio Ambiente, Geologia de Engenharia e Geotectônica. 